\title{
Developing and Evaluating Multi-Scale Map Styles: Creating a Multi-Scale Legend
}

\author{
Mathias Gröbe ${ }^{\mathrm{a},}$, Dirk Burghardt ${ }^{\mathrm{a}}$ \\ ${ }^{a}$ TU Dresden, Institute of Cartography, mathias.groebe@tu-dresden.de, Dirk Burghardt,dirk.burghardt@tu-dresden.de \\ * Corresponding author
}

Keywords: Multi-scale map design, zoomable web maps, multi-representation, multi-scale legend

\begin{abstract}
:
In recent years, the usage of zoomable maps strongly increased. The development of small and cheap electronic devices with wireless internet connection such as smartphones and tablets has made maps nowadays to a crucial part of everyday life. For the navigation and orientation, the user often uses zoomable maps (Muehlenhaus, 2014). Currently the technological development controls the map design and less cartographic design rules, which leads to the impress those current maps have a lower graphic quality. A sample for this trend is the new vector tile based maps, which offer the advantage of rotatable, multilingual maps build on one database (Martinelli and Roth, 2016).

There is a need for the development of cartographic design guidelines to guarantee consistent map readability over all zoom levels. The infinitely zoomable maps especially vector maps intensify this development. Analog maps had one specific scale, while a series of topographic maps were offered in manageable number of scales in comparison to OpenStreetMaps 20 zoom levels for raster tiles (Anon, 2019). Raster web maps had a number of predefined zoom levels, while vector maps offer the possibility of continuous zooming. This fact clarifies the requirement of research and development of rules for such types of maps.
\end{abstract}

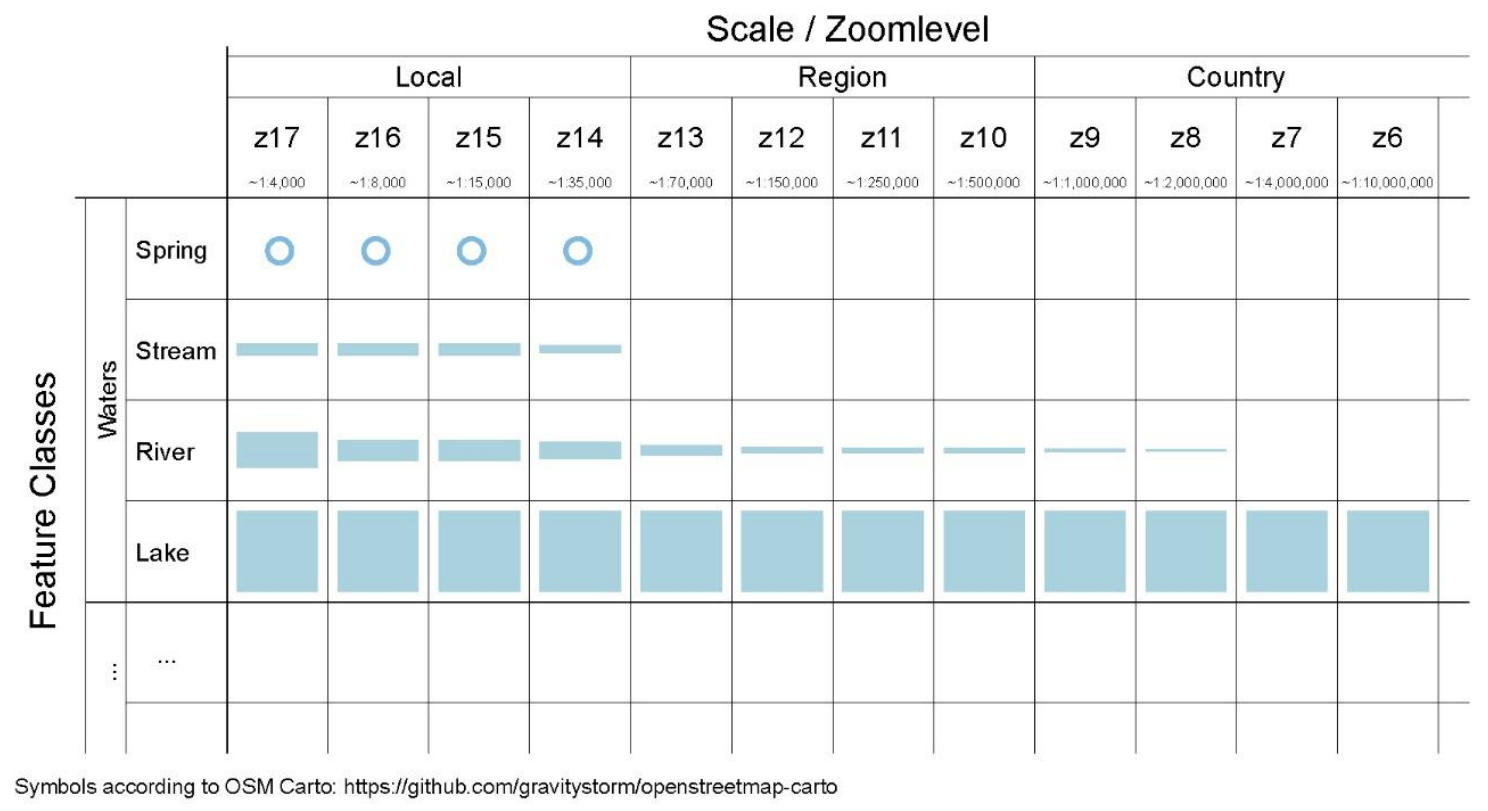

Figure 1. Example for a multi-scale legend using a low number of waters features symbols from the free available standard OpenStreetMap Carto style (Allan, 2019).

A first important task for the fulfilment of this objective is the evaluation of a multi-scale map styles. For this task, we developed the concept of a multi-scale legend. This new tool should help cartographers and designer to create, modify and improve multi-scale map styles. It can help to explore existing map styles, identify inconsistencies and support the design process. We decided to use the legend due to the abstraction of the map style from map content. For visualization of the scale-dependencies for each feature in a row, the scale/zoom level changes in each column. In combination with other map features and zoom levels results a two-dimensional matrix showing the scale-dependent visualization. This legend matrix shows the map features in every zoom level, which allows reaching an overview of the symbolization of features over several scales. In this way, it is possible to check how consistent a map style is in one zoom level as well as over a set of zoom levels. 
Figure 1 shows an example for a legend matrix using the OpenStreetMap Carto style: the representation of selected water bodies depending on the scale is illustrated. Streams and springs always occur together within the same zoom levels. In contrast to the rivers, the width of the streams remains nearly constant across the different scales. It is also visible that the color for the spring differs from the other water features. A multi-scale legend offers the possibility of grouping feature classes by topics (e.g. water bodies, vegetation and road network) as in the example. Other possibilities are geometry, color or occurrence in similar zoom levels. This can help in the search for errors, in the identification of breaks in the symbolization and in the development of continuous symbolization. The result is similar but more illustrative than the ScaleMaster (Brewer and Buttenfield, 2007, 2010), which is a diagram describing how feature classes are visualized depending from scale. Benefits of this legend are clarification of scale depended visualizations and the graphic implementation of design guidelines. A challenge is the implementation for different map styles due to the associated effort and the resulting sometimes very large overviews.

An on-going technological development takes place, wherefore cartographers should upgrade the design guidelines and methods for the production of current technological, well-looking maps. With the multi-scale legend, we provided a smart legend for a zoomable map. Nevertheless, these new ideas we have developed need more research and should always take the map purpose in account. Further, we would like to apply the multi-scale legend on existing map styles to reach more information about how these styles are working. In addition, we will further develop the multi-scale legend to a documentation of the creation of the map, showing how data is generalized and visualized.

\section{References}

Allan, A.: A general-purpose OpenStreetMap mapnik style, in CartoCSS: gravitystorm/openstreetmap-carto, CartoCSS. [online] Available from: https://github.com/gravitystorm/openstreetmap-carto (Accessed 2 April 2019), 2019.

Anon: Zoom levels - OpenStreetMap Wiki, [online] Available from: https://wiki.openstreetmap.org/wiki/Zoom_levels (Accessed 1 March 2019), 2019.

Brewer, C. A. and Buttenfield, B. P.: Framing Guidelines for Multi-Scale Map Design Using Databases at Multiple Resolutions, Cartography and Geographic Information Science, 34(1), 3-15, doi:10.1559/152304007780279078, 2007.

Brewer, C. A. and Buttenfield, B. P.: Mastering map scale: balancing workloads using display and geometry change in multi-scale mapping, GeoInformatica, 14(2), 221-239, doi:10.1007/s10707-009-0083-6, 2010.

Martinelli, L. and Roth, M.: Updatable Vector Tiles from OpenStreetMap, Hochschule für Technik Rapperswil, Rapperswil. [online] Available from:

https://eprints.hsr.ch/536/1/thesis_updatable_vector_tiles_from_openstreetmap.pdf, 2016.

Muehlenhaus, I.: Web cartography map design for interactive and mobile devices, CRC Press, Boca Raton., 2014. 